\title{
Predictive Value of Lateral Bending, Push-Prone, and Fulcrum-Bending Radiographs in Adolescent Idiopathic Scoliosis
}

\author{
Jose Joefrey F. Arbatin, Jr. ${ }^{1,2}$ and Rafael C. Bundoc ${ }^{2,3}$ \\ ${ }^{1}$ Cebu Orthopedic Institute, Cebu City \\ ${ }^{2}$ Department of Orthopedics, College of Medicine and Philippine General Hospital, University of the Philippines Manila \\ ${ }^{3}$ Department of Anatomy, College of Medicine, University of the Philippines Manila
}

\begin{abstract}
Introduction. The advent of pedicle screws which provide distraction and derotation has led to higher correction of major curves. Newer methods have been devised to evaluate preoperative coronal flexibility, including lateral-bending (LB), push-prone (PP) and fulcrum-bending (FB) radiographs. Documentation of a consistent radiographic method predictive of correction rate has not been established.
\end{abstract}

Objective. To determine the most predictive radiographic method for evaluating spine flexibility and correction by comparing the correction rate $(\mathrm{CR})$, flexibility rate $(\mathrm{FR})$ and correction index $(\mathrm{Cl})$ of the Cobb's angle using the different radiographic methods.

Methods. Preoperative radiographs of 20 patients who underwent spinal fusion for adolescent scoliosis were obtained using the LB, PP, and FB method and compared with postoperative radiographs.

Results. Comparing the mean Cobb angles using the different methods to that of postoperative standing showed that only the FB method is not significantly different from the latter $(p=0.669)$. There was significant difference between the Cobb's angle measured on the LB and PP and that measured on postoperative standing ( $p=0.043, p=0.008)$. Comparing the mean flexibility of the different methods with the mean $C R$ also showed that the mean FR of LB $(p=0.007)$ and PP $(p=0.00013)$ were significantly different from the $C R$ while that of $F B$ is not significantly different from the $C R(p=0.687)$.

Conclusion. The FB radiograph demonstrated no statistical difference compared to postoperative radiograph, $\mathrm{FR}$, and $\mathrm{Cl}$.

Key Words: scoliosis, radiography, adolescent, spinal fusion

Presented at the 23rd Annual Orthopedic Residents' Research Forum, December 2005, Philippine General Hospital.

Corresponding author: Jose Joefrey F. Arbatin, Jr.

Department of Orthopedics

Philippine General Hospital

University of the Philippines Manila

Taft Avenue, Ermita, Manila 1000 Philippines

Telephone: +632 5548466

Email: jefmd01@yahoo.com

\section{Introduction}

The assessment of the flexibility of the spine in patients with adolescent idiopathic scoliosis (AIS) is important because it provides information regarding the rigidity of the curve, extent of structural change, the levels to be included in the arthrodesis, the amount of correction that can be safely achieved, and whether a secondary curve should be treated with fusion. ${ }^{1}$

The advent of segmental spinal systems which provides distraction, translation, and derotation have led to higher postoperative correction of major curves. ${ }^{2}$ Thus, newer methods have been devised to evaluate preoperative coronal flexibility. These include supine, ${ }^{3}$ push-prone, ${ }^{4,5,6}$ traction, ${ }^{7}$ side-bending, 5,7,8,9,10 and fulcrum-bending radiographs. ${ }^{11,12}$

The goal of surgical management of scoliosis is to achieve a stable balanced spine centered over the pelvis. ${ }^{13}$ To determine the exact levels of fusion required to achieve this goal, surgeons have routinely used various types of preoperative radiographs to assess the flexibility of the spine. Currently, maximal side-bending supine radiographs are considered the gold standard for evaluating preoperative flexibility, as they have been found to be equal or better than other methods and are easily performed.,13,14,15,16 However, current segmental spinal instrumentation systems using pedicle screws, which are more rigid than the Harrington distraction system, have been found to achieve more correction than would be expected from the evaluation of traditional lateral-bending radiographs. ${ }^{12}$ For example, Aronsson has demonstrated the ineffectiveness of sidebending radiographs with the following results: sidebending, $22^{\circ}$ correction; Harrington instrumentation, $23^{\circ}$ correction; Wisconsin wires, 29० correction; and Texas Scottish Rite Hospital Instrumentation, $36^{\circ}$ correction. ${ }^{8}$ In addition, standardization of adequacy of side-bending radiographs is difficult and is partly dependent on active patient participation, effort and acceptance of the technique. ${ }^{10}$ It is particularly unreliable for patients with mental retardation or neuromuscular disorders. ${ }^{1}$

Kleinman et al. described the push-prone radiograph in which the physician applies manual pressure on the apices of each curve with the patient prone on the x-ray table. The average correction obtained for 82 curves was 21.1 degrees, as measured on the push films and 21.8 degrees 
postoperatively, which was not statistically significant. Furthermore, the close relationship between the push film and immediate postoperative correction was not altered by the location of the curve, the type of instrumentation employed, or the etiology of the scoliosis. ${ }^{5}$ Vedentam et al. compared the supine side-bending radiograph with the push-prone radiograph in terms of their ability to predict the postoperative coronal alignment for primary thoracolumbar and lumbar curves managed with an anterior spinal instrumentation. They concluded that the lateral bending and the push-prone radiographs predicted less correction of the Cobb angle than was achieved after surgery. However, the push-prone radiograph was superior to the lateralbending radiograph in accurately predicting the postoperative correction of the rotation of the lowest instrumented vertebra as well as translation of the lowest instrumented vertebra from the sacral line. ${ }^{6}$ They noted the advantage of the push-prone radiograph in its ability to assess the effect of forceful correction of the primary compensatory curve on the curves above and below the fusion. This allows the surgeon to determine before surgery the eventual global coronal balance of the spine after correction of the primary curve. ${ }^{6}$ Dobbs et al. tried to determine the best preoperative radiograph which correlates with the postoperative lumbar curve in AIS patients undergoing a selective thoracic posterior spinal fusion at L1 or above. ${ }^{14}$ The study evaluated 100 patients with AIS and compared side-bending and push-prone radiographs. Results showed that when trying to predict ultimate lumbar spine position using all available pre-op measures in a multiple linear regression model, upright AP lumbar Cobb $(\mathrm{p}<0.0001)$ and push-prone lumbar Cobb $(\mathrm{p}<0.03)$ were the only pre-op measures predictive of final lumbar Cobb measurement. ${ }^{14}$

In an effort to provide maximal flexibility with a technique that is simple to perform and does not require active patient participation, Luk and Cheung et al. devised the fulcrum-bending radiograph designed to provide true reproducibility by correcting both gravitational and postural forces. The study revealed that the difference between the mean angle on the lateral bending radiograph and that of the postoperative radiograph was significant $(\mathrm{p}<0.001)$. However, the mean angle measured on the preoperative fulcrum bending radiograph and the postoperative angle were almost identical. ${ }^{11,12}$ They attributed this to the passive bending force exerted on the curve by the body weight, and its reproducibility, with no muscular effort. Klepps et al. compared supine side-bending and fulcrum-bending radiographs in assessing coronal flexibility in 46 patients with AIS. ${ }^{10}$ The study compared the absolute values, flexibility rates, correction rates, and correction indices of the Cobb's angles and showed that the fulcrum-bending radiograph demonstrated statistically better correction than other preoperative methods of main thoracic curves $(p<0.01)$ but fell short of demonstrating the correction obtained surgically. There was no statistical difference between sidebending, fulcrum-bending, or postoperative correction for thoracolumbar/lumbar curves (all $\mathrm{p}>0.07){ }^{10}$

Deviren et al. reported that the flexibility of the major curve is a useful predictor of expected surgical correction, and flexibility of compensatory curves determines whether they are structural or nonstructural. ${ }^{3}$ They determined that for every $10^{\circ}$ increase in curve magnitude over $40^{\circ}$ results in a $10^{\circ}$ decrease in flexibility; every 10 year increase in age decreases flexibility of the structural curve by $5 \% .^{3}$ They advocated further studies because a quantitative assessment of changes in curve flexibility with age and progression of deformity may yield important insight into the change in surgical management options over time.

This study presents the first attempt in Philippine medical literature to analyze the correlation of lateralbending, push-prone and fulcrum-bending radiographs in terms of their ability to assess coronal flexibility and postoperative alignment for primary thoracolumbar and lumbar curves managed with posterior spinal instrumentation. In addition, the study will determine the role of age and flexibility of the spine in predicting the correction rate.

\section{Methods}

All patients with AIS who underwent posterior instrumentation with pedicle screws from December 2004 to October 2005 were included in the study having fulfilled the inclusion criteria:

1. Detection of curve after the age of 10 years

2. Absence of congenital, musculoskeletal, and syndrome-like causes.

3. Complete treatment by the Spine Service

4. Posterior instrumentation and fusion utilizing pedicle screws

5. Complete radiographic evaluation.

Written consent for the radiography was obtained from all the parents or guardians. The preoperative radiologic evaluation for all patients included a 36-inch long posteroanterior radiograph taken with the patient standing, a supine posteroanterior radiograph, a supine right and left side-bending posteroanterior radiograph, a push-prone radiograph, and a fulcrum-bending radiograph. Sidebending radiographs were taken with the patients voluntarily bending maximally while in a supine position (Figure 1). ${ }^{10}$ The push-prone radiograph was obtained by positioning the patient comfortably in a prone position on a 36-inch long cassette. A trained radiology technician then applied a firm medial translational force to the portion of the torso that corresponds to the apex of the primary curve. ${ }^{5}$ Counter forces were applied to the opposite side of the body of the axilla and the pelvis by a second assistant, thereby creating a three-point correctional force on the spine. 


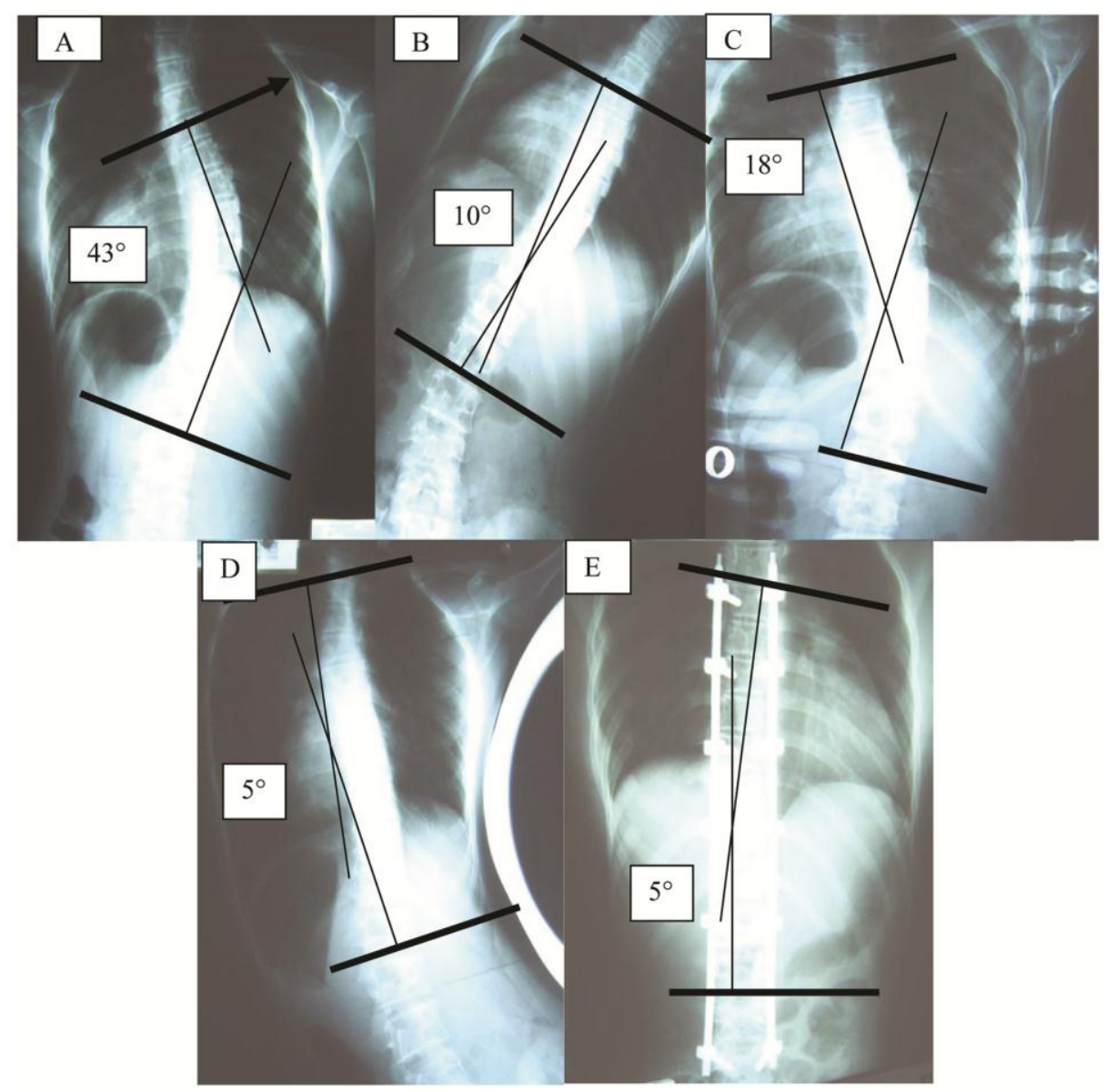

Figure 1. S. A. is a 13-year-old girl who has a primary thoracolumbar curve, with the superior end vertebra being T6 and the lower end vertebra being L2. (A) The standing radiograph demonstrated a $43^{\circ}$ right thoracic curve; (B) The lateral bending radiograph demonstrated a $10^{\circ}$ curve while the $(\mathrm{C})$ push prone radiograph resulted in an $18^{\circ}$ curve; (D) The fulcrum bending radiograph showed a $5^{\circ}$ curve; (E) The postoperative standing radiograph showed the curve corrected to $5^{\circ}$.

Accurate placement of the apical force on the torso was performed by identifying the apex of the curve on the standing posteroanterior radiograph and then marking the apex on the patient's torso.

A fulcrum-bending radiograph was made with the patient lying on his/her side over a large $(23 \mathrm{~cm}$ in diameter), radiolucent plastic cylinder. The fulcrum was placed directly under the apex of the lumbar curve and under the rib corresponding to the apex of the thoracic curve. Accurate placement of the fulcrum was achieved by identifying the apex of the curve and its corresponding rib on the anteroposterior radiograph and then marking the apex on the patient after counting cephalad from the most caudad rib. A true lateral position of the patient on the table was determined with reference to the pelvis and the shoulder, which should be perpendicular to the $\mathrm{x}$-ray beam. ${ }^{11,12}$

The postoperative radiographic evaluation was taken one week after surgery with a standing posteroanterior view of the spine using a 36-inch long cassette.
The Cobb method was used to measure the thoracolumbar or lumbar curves. The Cobb method consisted of three steps: (1) locating the superior end vertebra, (2) locating the inferior end vertebra, and (3) drawing intersecting perpendicular lines from the superior surface and from the inferior surface of the inferior end vertebra. The angle of deviation of these perpendicular lines from a straight li ne is the angle of the curve. Measurements on all radiographs were made by the author independently confirmed by a radiology resident. The average of the values will be used when two different values are observed for the same parameter. A number 2 glass marking lead pencil sharpened to a 2-mm tip and a standard goniometer with $1^{\circ}$ increments were used to determine the measurements. Carman et al. determined the intraobserver and interobserver variation in the measurement of the Cobb's angle to be $1.06 \pm 2.97$ and $3.9 \pm 3.1$ degrees, respectively. ${ }^{1}$ 
The Cobb angles were compared by their absolute values, flexibility rates, correction rates, and correction indices using the following formulas:

Correction rate $=\underline{\text { Preoperative standing } \mathrm{Cobb} \text { angle }- \text { Postoperative } \mathrm{Cobb} \text { angle }} \times 100$

Preoperative standing Cobb angle

Flexibility rate $=\underline{\text { Preoperative standing } \mathrm{Cobb} \text { angle }- \text { Preopeartive flexibility angle }} \times 100$ Preoperative standing Cobb angle

$$
\text { Correction Index }=\frac{\text { Correction Rate }}{\text { Flexibility Rate }}
$$

transform the scoliosis into a kyphosis at the thoracic spine and into a lordosis at lumbar spine and restore the sagittal profile

All surgery was performed by a single surgeon (RCB). All of the patients were managed with posterior spinal arthrodesis with the use of pedicle screws (Moss-Miami instrumentation or Sofamor Danek). Levels of instrumentation were selective and based on curve classification as advocated by King and Moe. ${ }^{17} \mathrm{~A}$ rod derotation technique was utilized for curve correction wherein the correction rod is rotated 90 degrees to transform the scoliosis into a kyphosis at the thoracic spine and into a lordosis at the lumbar spine and to restore the sagittal profile. ${ }^{18,19}$ Thereafter, a Stagnara wake up test was performed to document voluntary distal motor function. Statistical evaluation utilized paired $t$ test using Microsoft Excel statistical program to determine the $p$ values. Values less than 0.05 were considered statistically significant. Predictors of correction rate were evaluated. Pearson correlation and multilinear regression analysis using Statistical Analysis System for Windows software (ver.6.12) were used to determine correlations and relationships between the variables.

\section{Results}

During the period of December 2004 - October 2005, a total of 22 patients with adolescent idiopathic scoliosis who underwent posterior instrumentation were seen at the Spine Unit. One (1) patient had incomplete radiographs while one (1) patient had a congenital type of scoliosis; these patients were thus excluded from the study. This left a total of 20 patients who fulfilled the inclusion criteria forming the population of this study.

The study included 15 female patients with an average age of 16.4 years (range, 12-22) at the time of surgery. The patients were divided into groups according to the five curve patterns described by King. ${ }^{17}$ Two (2) patients had a Type I curve, six (6) patients had a type II curve, three (3) patients had a type III curve, eight (8) had a type IV curve and one (1) had a type V curve. Table 1 shows that the mean preoperative Cobb angle that was measured on the preoperative standing radiograph was $55^{\circ} \pm 14\left(\right.$ range $34-85^{\circ}$ ).

\begin{tabular}{|c|c|c|c|c|c|c|c|c|}
\hline \multirow[t]{2}{*}{ Px\# } & \multirow{2}{*}{$\begin{array}{c}\text { Age at } \\
\text { operation }\end{array}$} & \multirow{2}{*}{$\begin{array}{c}\text { Type of } \\
\text { curve }\end{array}$} & \multicolumn{5}{|c|}{ Angle of Curve (degrees) } & \multirow[b]{2}{*}{ correction rate ${ }^{*}$} \\
\hline & & & $\begin{array}{c}\text { Preop } \\
\text { standing }\end{array}$ & Side-bend & Push-prone & $\begin{array}{l}\text { Fulcrum } \\
\text { bending }\end{array}$ & $\begin{array}{c}\text { Post-op } \\
\text { standing }\end{array}$ & \\
\hline 1 & 19 & V & 60 & 58 & 54 & 40 & 48 & 20 \\
\hline 2 & 22 & II & 58 & 28 & 30 & 12 & 14 & 76 \\
\hline 3 & 15 & I & 60 & 38 & 35 & 18 & 15 & 75 \\
\hline 4 & 21 & IV & 70 & 30 & 38 & 24 & 25 & 64 \\
\hline 5 & 16 & III & 55 & 35 & 32 & 22 & 22 & 60 \\
\hline 6 & 14 & IV & 45 & 6 & 18 & 4 & 4 & 91 \\
\hline 7 & 14 & I & 34 & 8 & 19 & 5 & 4 & 88 \\
\hline 8 & 15 & IV & 85 & 65 & 60 & 56 & 50 & 41 \\
\hline 9 & 14 & IV & 76 & 60 & 33 & 30 & 8 & 89 \\
\hline 10 & 13 & III & 43 & 10 & 18 & 5 & 5 & 88 \\
\hline 11 & 21 & II & 75 & 50 & 65 & 50 & 33 & 56 \\
\hline 12 & 14 & IV & 54 & 35 & 35 & 23 & 20 & 63 \\
\hline 13 & 15 & II & 45 & 10 & 12 & 8 & 6 & 87 \\
\hline 14 & 16 & II & 48 & 19 & 24 & 15 & 18 & 62 \\
\hline 15 & 15 & II & 40 & 18 & 18 & 12 & 10 & 75 \\
\hline 16 & 19 & II & 45 & 22 & 24 & 15 & 15 & 67 \\
\hline 17 & 15 & III & 35 & 12 & 18 & 12 & 15 & 57 \\
\hline 18 & 18 & IV & 55 & 22 & 28 & 20 & 20 & 64 \\
\hline 19 & 14 & IV & 60 & 28 & 30 & 20 & 24 & 58 \\
\hline 20 & 16 & IV & 55 & 24 & 24 & 18 & 16 & 71 \\
\hline Mean & 16 & & 58 & 33 & 34 & 22 & 19 & 69 \\
\hline SD & 3 & & 14 & 20 & 16 & 17 & 15 & 21 \\
\hline
\end{tabular}

Table 1. Case-specific data from 20 patients

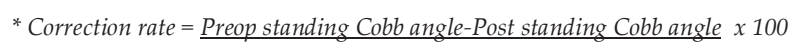

Preop standing Cobb angle 
Table 2. Average Cobb's angle, flexibility rate and correction index

\begin{tabular}{lccccc}
\hline & Preoperative Standing & Lateral-Bending & Push-Prone & Fulcrum-Bending & Postoperative standing \\
\hline $\begin{array}{l}\text { Cobb's Angle } \\
\text { mean(SD) }\end{array}$ & $58.46(14.88)$ & $33.31(20.71)$ & $34.54(16.57)$ & $22.85(17.17)$ & $19.54(15.86)$ \\
Flexibility Rate & & & & & \\
Correction IndexII & n/a & $46.54(25.11)$ & $42.85(18.02)$ & $64.46(20.89)$ & n/a \\
\hline
\end{tabular}

¥ Standard Deviation

*Flexibility rate $=\underline{\text { Preop standing Cobb angle-Preop flexibility angle }} \times 100$ Preop standing Cobb angle

II Correction Index $=\underline{\text { Correction Rate }}$ Flexibility Rate

Note: Correction indices designed to factor out curve flexibility were calculated. A value of 1 indicates a flexibility rate similar to the surgical correction rate.

Table 2 lists the mean Cobb angle, flexibility rate and correction index of the preoperative standing, lateralbending, push-prone, fulcrum-bending and the postoperative standing. Comparing the mean Cobb angles using the different methods to that of the postoperative standing using T-test showed that only the fulcrum bending method is not significantly different from the latter $(p=0.159)$. There were significant differences between the Cobb's angle measured on the lateral-bending and pushprone and that measured on the postoperative standing $(\mathrm{p}=0.003, \mathrm{p}=0.000008)$ (Table 3).

Table 3. Comparison of mean Cobb's angle of lateralbending, push-prone, and fulcrum-bending vs. postoperative Cobb's angle

\begin{tabular}{lll}
\hline \multicolumn{1}{c}{ Radiographic method } & $\begin{array}{c}\text { Cobb's angle } \\
\text { Mean (SD) }\end{array}$ & P value \\
\hline Lateral-bending & $33.31(20.71)$ & 0.003 \\
Push-prone & $34.54(16.57)$ & 0.000008 \\
Fulcrum-bending & $22.85(17.17)$ & 0.159 \\
Postoperative standing & $19.54(15.86)$ & N/A \\
\hline
\end{tabular}

The mean correction rate obtained by the study was 69.07 with standard deviation of 21.43. The Pearson correlation coefficient when the correction rate was correlated with the flexibility rate of lateral-bending, pushprone and fulcrum-bending are 0.762, 0.856 and 0.868, respectively. Table 4 shows that the fulcrum-bending method has the strongest correlation with the correction rate. Figure 2 shows the scatter plot of the flexibility rate of fulcrum-bending indeed which shows a strong positive correlation with the correction rate. T-test comparing the mean flexibility of the different methods with the mean correction rate also showed the same result, that is, the mean flexibility rates of lateral-bending $(p=0.0003)$ and push-prone $(\mathrm{p}=0.000002)$ were significantly different from the correction rate while that of fulcrum-bending is not significantly different from the correction rate $(\mathrm{p}=0.15)$ (Table 4).

A correction index of 1 or more suggests that the instrumentation has taken up or exceeded nearly all the flexibility revealed by the fulcrum-bending radiograph. The mean correction index of the fulcrum-bending method is the closest to 1 and not significantly different $(p=0.205)$ from 1 while that of the lateral-bending $(\mathrm{p}=0.038)$ and push-prone $(p=0.003)$ methods were significantly different (Table 5).

Table 4. Comparison of flexibility rate of lateral-bending, push-prone, and fulcrum-bending vs. correction rate

\begin{tabular}{lccl}
\hline & $\begin{array}{c}\text { Flexibility } \\
\text { Rate }\end{array}$ & $\begin{array}{c}\text { Pearson correlation } \\
\text { coefficient }\end{array}$ & P value \\
\hline Lateral bending & $46.54(25.11)$ & 0.762 & 0.0003 \\
Push prone & $42.85(18.02)$ & 0.856 & 0.0000002 \\
Fulcrum bending & $64.46(20.89)$ & 0.868 & 0.152 \\
\hline
\end{tabular}

Table 5. Comparison of correction index of lateral-bending, push-prone, and fulcrum-bending

\begin{tabular}{llc}
\hline & Correction Index & P value \\
\hline Lateral bending & $2.06(1.64)$ & 0.038 \\
Push prone & $1.82(0.78)$ & 0.003 \\
Fulcrum bending & $1.10(0.26)$ & 0.205 \\
\hline
\end{tabular}

Multilinear regression analysis was utilized to explain the association between correction rate, flexibility rate of fulcrum bending and age (Figure 2). Statistical analysis demonstrated a linear association predicted by the formula:

Correction rate $=33.864-1.086$ (age) +0.815 (flexibility rate of fulcrum bending)

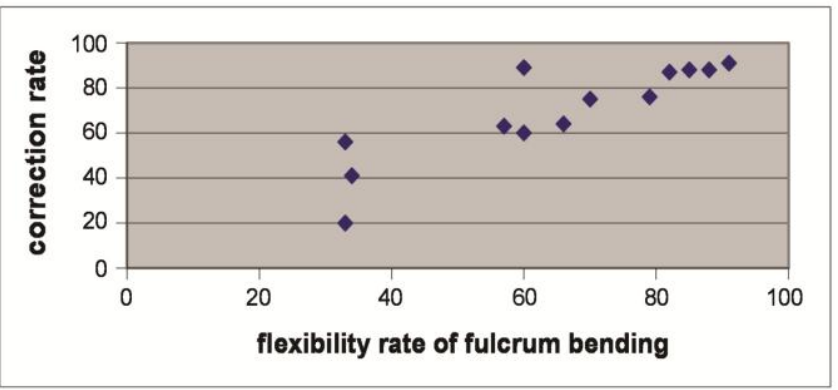

Figure 2. Scatter plot of flexibility rate of fulcrum bending and correction rate 
Thus, the correction rate decreases by around 1\% for every one-year increase in age when the flexibility rate is held constant. Similarly, maintaining the age constant, the correction rate increases by around $0.8 \%$ for every $1 \%$ increase in the flexibility rate.

\section{Discussion}

From December 2004 to October 2005, the Spine Unit of the Department of Orthopedics treated 22 patients with adolescent idiopathic scoliosis. A total of 20 patients fulfilled the inclusion criteria and form the population for this study. The study presents the first attempt in Philippine medical literature to correlate lateral-bending, push-prone and fulcrum-bending radiographs with the correction rate, flexibility rate and correction index. Several similarities and differences are observed in comparison with regional and international reports.

Currently, lateral bending radiographs have been the standard by which other flexibility radiographs are compared. However, several authors have called into question their ability to provide correction comparable to that obtained through modern spinal instrumentation. ${ }^{1,5,8,11,20,21}$ For example, Aronsson has demonstrated the ineffectiveness of side-bending radiographs with the following results: side-bending, $22^{\circ}$ correction; Harrington instrumentation, $23^{\circ}$ correction; Wisconsin wires, $29^{\circ}$ correction; and Texas Scottish Rite Hospital Instrumentation, $36^{\circ}$ correction. $^{8}$ Also, standardization of adequacy of side-bending radiographs is difficult and is partly dependent on patient participation and acceptance of the technique. Cheung et al. reported that the difference between the mean angle on the lateral-bending radiograph and that on the postoperative radiograph was significant $(p<0.001){ }^{11,12}$ In our study there was significant difference between the Cobb's angle of the lateral-bending compared to the postoperative standing radiograph $(p=0.043)$. Furthermore, there was a significant difference between the flexibility rate of the lateral-bending radiograph and the correction rate $(\mathrm{p}=0.007)$.

The push-prone technique assesses overall spinal balance by demonstrating the effect of primary curve correction on the curves both above and below and exposes patients to less radiation, but when compared to lateralbending, predicts less correction. ${ }^{4,5,6}$ Vendantam et al. reported in a prospective study of 40 patients with adolescent idiopathic scoliosis that there was significant difference in the Cobb's angle measured in the push-prone radiograph and the postoperative radiograph $(p=0.0086)$. They attributed their results to the difficulty in standardizing the force exerted on the apex of the curve. ${ }^{6}$ Klepps et al. compared the correction index obtained with a push-prone radiograph and determined that it was significantly different from a fulcrum-bending radiograph $(\mathrm{p}$ $<0.05) .{ }^{10}$ The current study shows that the push-prone radiographs were significantly different from the postoperative standing radiographs $(\mathrm{p}=0.008)$, flexibility rate $(p=0.00013)$ and correction index $(p=0.001)$.

In an effort to provide maximal flexibility with a technique that is simple to perform and does not require active patient participation, Luk et al. devised the fulcrumbending radiograph designed to provide true reproducibility by correcting both gravitational and postural forces. Our study has confirmed the results of these previous studies which found fulcrum-bending radiographs demonstrating better flexibility than other preoperative methods. ${ }^{11,12}$ The study of Klepps showed that fulcrumbending radiographs provided significantly better correction than lateral bending and push prone in terms of average Cobb's angle, flexibility rate, or correction index (all $\mathrm{p}<0.01) .{ }^{10}$ Cheung reported that even in patients who had a more flexible curve, the difference between the angle measured on the lateral-bending radiograph and that on the fulcrum-bending radiograph was still significant $(\mathrm{p}<0.01) .{ }^{11}$ Furthermore, the difference between the mean angle measured using the fulcrum-bending radiograph and that using the postoperative radiograph was 1 degree. Their conclusion was that flexibility is best predicted by the fulcrum-bending radiograph and is always more predictive of the final correction. In their institution, the fulcrumbending radiograph has replaced the lateral-bending radiograph in routine preoperative assessment. In our study, the fulcrum-bending radiograph showed no significant difference compared to postoperative standing radiograph $(p=0.669)$, flexibility rate $(p=0.687)$, and correction index $(\mathrm{p}=0.816)$.

Deviren et al. reported that flexibility of the major curve is a useful predictor of expected surgical correction, and flexibility of compensatory curves determines whether they are structural or nonstructural. ${ }^{3}$ They determined that for every $10^{\circ}$ increase in curve magnitude over $40^{\circ}$ results in a $10^{\circ}$ decrease in flexibility; every 10 year increase in age decreases flexibility of the structural curve by $5 \% .{ }^{8}$ In our study, age and fulcrum flexibility rate were the main predictors for correction rate. Every one-year increase in age when the flexibility rate is held constant, results in a $0.9 \%$ decrease in the correction rate. Similarly, maintaining the age constant, the correction rate increases by around $0.8 \%$ for every $1 \%$ increase in the flexibility rate.

\section{Conclusion}

The fulcrum-bending radiograph demonstrated no statistical difference as compared to the postoperative radiograph, flexibility rate, and correction index. In our institution, fulcrum-bending radiographs have been a standard procedure before surgery because of its ability to assess postoperative curve correction. Age and fulcrum flexibility rate were the main predictors for correction rate. Every one-year increase in age when the flexibility rate is held constant results in a $0.9 \%$ decrease in the correction rate. 


\section{References}

1. Carman DL, Browne RH, Birch JG. Measurement of scoliosis and kyphosis Radiographs. Intraobserver and interobserver variation. J Bone Joint Surg Am. 1990; 72(3):328-33.

2. Morrissy RT, Goldsmith GS, Hall EC, Kehl D, Cowie GH. Measurement of the Cobb angle on radiographs of patients who have scoliosis. Evaluation of intrinsic error. J Bone Joint Surg Am. 1990; 72(3):320-7.

3. Deviren V, Berven S, Kleinstruck F, Antinnes J, Smith JA, Hu SS. Predictors of flexibility and pain patterns in thoracolumbar and lumbar idiopathic scoiliosis. Spine. 2002; 27(21):2346-9.

4. Duval-Beaupère G, Lespargot A, Grossiord A. Flexibility of scoliosis: What does it mean? Is this terminology appropriate? Spine. 1985; 10(5):428-32.

5. Kleinmann RG, Csongradi JJ, Rinsky LA, Bleck EE. The radiographic assessment of spinal flexibility in scoliosis: a study of the efficacy of the prone push film. Clin Orthop Relat Res. 1982; 162:47-53.

6. Vedantam R, Lenke LG, Bridwell KH, Linville DL. Comparison of pushprone and lateral-bending radiographs for predicting postoperative coronal alignment in thoracolumbar and lumbar scoliotic curves. Spine. 2000; 25(1):76-81.

7. Polly DW Jr, Sturm PF. Traction versus supine side bending. Which technique best determines curve flexibility? Spine. 1998; 23(7):804-8.

8. Aronsson DD, Stokes IA, Ronchetti PJ, Richards BS. Surgical correction of vertebral axial rotation in adolescent idiopathic scoliosis: prediction by lateral bending films. J Spinal Disord. 1996; 9(3):214-9.

9. Klepps SJ, Lenke LG, Bridwell KH, Bassett GS, Whorton J. Prospective comparison of flexibility radiographs in adolescent idiopathic scoliosis. Spine. 2001; 26(5):E74-9.

10. Cheung KM, Luk KD. Prediction of correction of scoliosis with use of the fulcrum bending radiograph. J Bone Joint Surg Am. 1997; 79(8):114450 .

11. Luk KD, Cheung KM, Leong JC. Assessment of scoliosis correction in relation to flexibility using the fulcrum bending correction index. Spine. 1998; 23(21):2303-7.

12. Richards BS, Birch JG, Herring JA, Johnston CE, Roach JW. Frontal plane and sagittal plane balance following Cotrel-Dubousset instrumentation for idiopathic scoliosis. Spine. 1989; 14(7):733-7.

13. Dobbs MB, Lenke LG, Bridwell K. Can we predict the ultimate lumbar curve in adolescent idiopathic scoliosis patients undergoing a selective thoracic fusion? Abstract from the SRS 2002 Annual Meeting.

14. Large DF, Doig WG, Dickens DR, Torode IP, Cole WG. Surgical treatment of double major scoliosis: improvement of the lumbar curve after fusion of the thoracic curve. J Bone Joint Surg Br. 1991; 73(1):121-4.

15. McCall RE, Bronson W. Criteria for selective fusion in idiopathic scoliosis using Cotrel-Dubousset instrumentation. J Pediatr Orthop. 1992; 12(4):475-9.

16. Bryd JA 3rd, Scoles PV, Winter RB, Bradford DS, Lonstein JE, Moe JH Adult idiopathic scoliosis treated by anterior and posterior spinal fusion. J Bone Joint Surg Am. 1987; 69(6):843-50.

17. King HA, Moe JH, Bradford DS, Winter RB. The selection of fusion levels in thoracic idiopathic scoliosis. J Bone Joint Surg Am. 1983; 65(9):1302-13.

18. Cotrel Y, Dubousset J, Guillaumat M. New universal instrumentation in spinal surgery. Clin Orthop Relat Res. 1998; 227:10-23.

19. Moe JH. Methods of correction and surgical techniques in scoliosis Orthop Clin North Am. 1972; 3(1):17-48

20. White AA, Panjab MM. Clinical Biomechanics of the Spine, $2^{\text {nd }}$ ed Philadelphia: JB Lippincott; 1990. pp. 138-143.

21. Wojcik AS, Webb JK, Burwell RG. An Analysis of the effect of the Zielke operation on S-shaped curves in idiopathic scoliosis. Spine. 1989; 14(6):6256-31

\section{PHILIPPINE BOARD OF ORTHOPAEDICS}
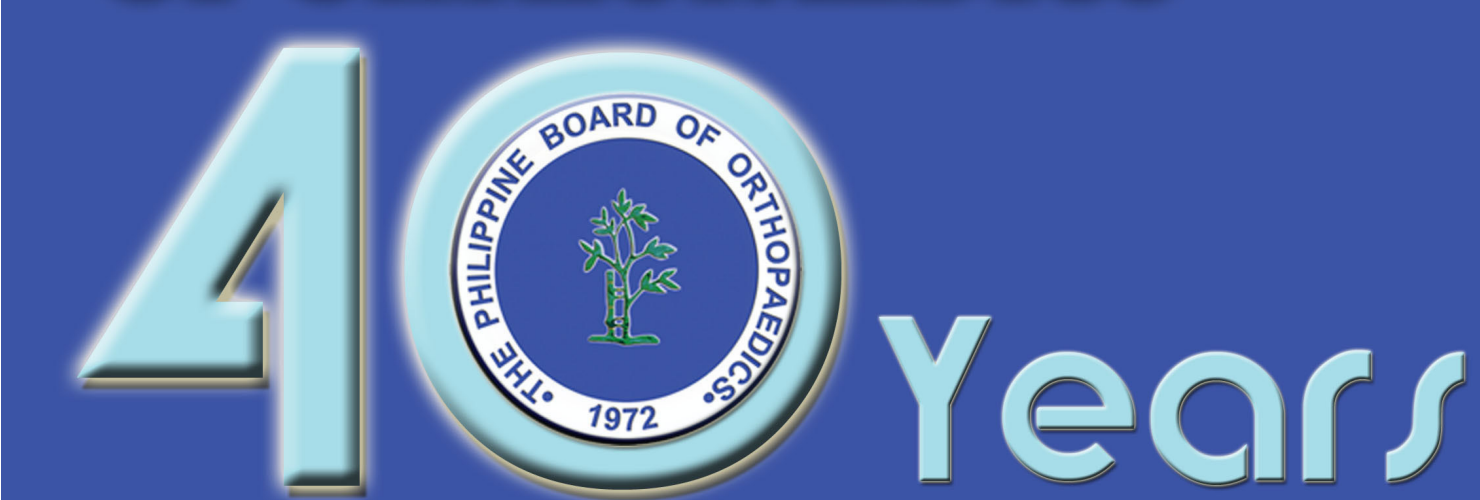

Philippine Orthopaedic Association

63rd Annual Convention

Nov. 14-17, 2012

Crowne Plaza Manila Galleria

ADB Corner Ortigas Ave.

Quezon City, Philippines 